\title{
Transition Analysis for the Mars Science Laboratory Entry Vehicle
}

\author{
Chau-Lyan Chang ${ }^{*}$, Meelan M. Choudhari ${ }^{*}$, Brian R. Hollis ${ }^{* *}$, and Fei Li ${ }^{*}$ \\ NASA Langley Research Center, Hampton, VA23681
}

\begin{abstract}
Viscous Laminar-turbulent transition plays an important role in the design of the Mars Science Laboratory (MSL) entry vehicle. The lift-to-drag ratio required for the precision landing trajectory will be achieved via an angle of attack equal to $\mathbf{1 6}$ degrees. At this relatively high angle of attack, the boundary layer flow near the leeward meridian is expected to transition early in the trajectory, resulting in substantially increased heating loads. This paper presents stability calculations and transition correlations for a series of wind tunnel models of the MSL vehicle. Experimentally measured transition onset locations are used to correlate with the N-factor calculations for various wind tunnel conditions. Due to relatively low post-shock Mach numbers near the edge of the boundary layer, the dominant instability waves are found to be of the first mode type. The $\mathbf{N}$-factor values correlating with measured transition onset at selected test points from the Mach 6 conventional facility experiments fall between 3.5 and 4.5 and apparently vary linearly with the wind tunnel unit Reynolds number, indicating strong receptivity effect. The small transition $\mathrm{N}$ value is consistent with previous correlations for second-mode dominant transition in the same wind tunnel facility. Stability calculations for stationary and traveling crossflow instability waves in selected configurations indicate that an $\mathrm{N}$ value of 4 and 6 , respectively, correlates reasonably well with transition onset discerned from one experimentally measured thermographic image.
\end{abstract}

\section{Introduction}

$\mathrm{T}$ he Mars Science Laboratory (MSL) vehicle is scheduled to launch in 2011. For the first time, a precision landing will be attempted for scientific payloads for Mars missions. In the current design, a controlled trajectory during the entry can be accomplished with a prescribed lift-to-drag ratio of 0.24 , which is achieved via an angle of attack equal to 16 degrees [1]. Similar to the previous Viking or Pathfinder missions, the basic configuration under consideration is a 70-degree sphere-cone forebody with a conic or biconic aftbody. However, due to larger payloads, the MSL vehicle will be both larger and heavier than its predecessors. To achieve the design goal, accurate aerodynamic and aerothermodynamic predictions of the vehicle performance become a crucial step for the success of the mission.

Accurate characterization of laminar turbulent transition is required to enable reliable aerothermodynamic predictions for the MSL entry vehicle. At its high angle-of-attack, the flow over the leeward side of MSL forebody is expected to become turbulent early in the flight trajectory. Because of the larger size of the MSL vehicle in comparison with previous missions to Mars, turbulent surface heating will be much more significant. Several computational and experimental investigations of the Mars entry vehicle have been performed during the past few years [2-4].

Transition measurements have been performed for an MSL model in the NASA Langley Research Center 20-inch Mach 6 Air Tunnel and in the Calspan, Univeristy of Buffalo (CUBRC) Large-Energy National Shock (LENS) $\mathrm{CO}_{2}$ Tunnel [1]. Due to the limited scope, only the Mach 6 Air Tunnel results are analyzed in this paper. Development of instrumentation that is planned to be included on the MSL flight vehicle is under way. Detailed stability and transition computations can help provide useful information concerning measurements of the transition onset on the vehicle surface. In spite of the 70-degree sphere-cone design of the flight vehicle, both surface heat transfer and thermographic phosphor image measurements were performed for several models with different cone angles in the

\footnotetext{
${ }^{*}$ Aerospace Technologist, Computational Aerosciences Branch

*** Aerospace Technologist, Aerothermodynamics Branch
} 
Mach 6 wind tunnel. The main objective of the present investigation is to perform preliminary computational studies of laminar-turbulent transition on blunt body flows in general, and on a series of the MSL vehicle models in particular. Physics-based transition prediction using both traditional linear stability theory (LST) and the more advanced parabolized stability equations (PSE) method are used for transition analyses of the selected wind-tunnel configurations. Results from the present investigation for different wind tunnel MSL models may enhance current understanding of the blunt body transition flow physics and improve transition prediction capability for future entry vehicles. In the text that follows, the MSL configurations and meanflow computations will be discussed in Section 2 , followed by a meanflow grid-convergence study in Section 3. Detailed stability results for most of the cases from the wind tunnel test matrix will be described in Section 4. A brief summary will be given at the end.

\section{Mars Science Laboratory Configurations and Meanflow Computations}

The current MSL entry vehicle configuration has a 70-deg sphere-cone forebody and a biconic aftbody with semi angles of 37-deg and 59-deg, respectively [1]. The maximum base radius is about $2.25 \mathrm{~m}$ and nose radius is half of that value. It is both larger and heavier than its predecessors, Viking, Pathfinder, or Mars Exploration Rover (MER). The wind tunnel models tested in the 20-In Mach 6 Langley air tunnel are much smaller scale models. Three different forebody configurations with 50-deg, 60-deg, and 70-deg sphere-cone geometries as shown in Fig. 1 were tested. The base diameter was equal to $15.24 \mathrm{~cm}$; however, an additional base diameter of $17.78 \mathrm{~cm}$ was also employed for the 70-deg configuration. The unit Reynolds number varied from $3.95 \times 10^{6}$ to $7.3 \times 10^{6}$ per foot.

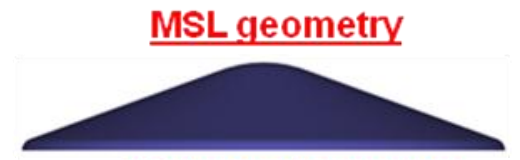

70-deg sphere-cone

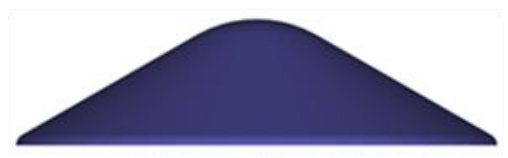

60-deg sphere-cone

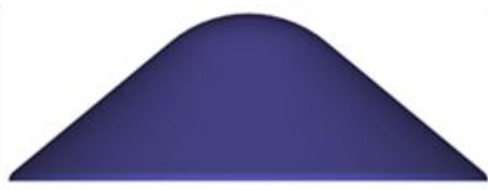

50-deg sphere-cone

Figure 1 Three sphere-cone MSL wind tunnel models (afterbody not shown) tested at NASA Langley Research Center

Surface heating rate and transition onset were measured in the wind tunnel experiments using a global thermographic phosphor system. Figures 2 and 3 show examples of the measured surface heating distributions mapped on the model surfaces for the 50-deg and 60-deg sphere-cone models at an angle of attack $\alpha$ of $16^{\circ}$ and various Reynolds numbers. The high-intensity nearly circular shape regions slightly below the center are associated with the laminar stagnation heating around the nose. Windward and leeward sides are below and above the stagnation regions, respectively. High surface heating levels on the leeward side surface indicate possible turbulent flows. Transition fronts marked by the upstream boundaries of the high-intensity turbulent regions in the 50-deg sphere-cone are mostly off the symmetry meridian, indicating possible crossflow instability. On the contrary, the two middle Reynolds number cases (Figs. 3(b) and 3(c)) of the 60-deg model have a clear symmetry meridian transition onset. Stability results for some of these configurations that can help explain these two different transition patterns will be discussed in the next section.

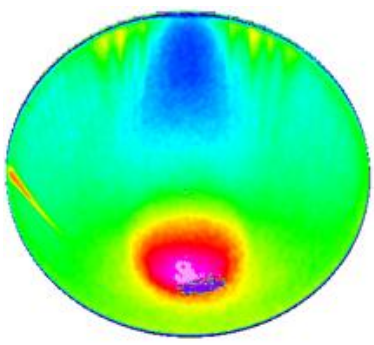

(a)

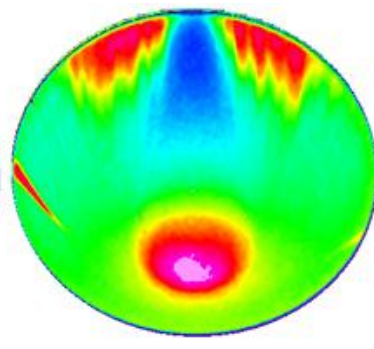

(b)

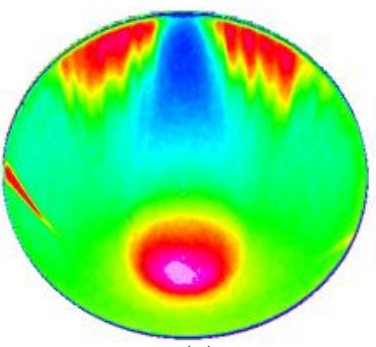

(c)

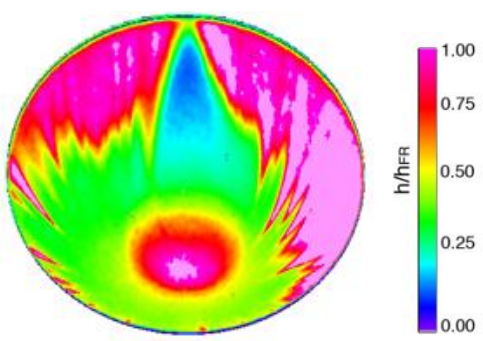

(d)

Figure 2 Distribution of surface heating levels from experimental theomographic images for 50-deg spherecone wind tunnel models at 16 degree angle of attack and four unit Reynolds numbers: (a) 3.95 x $10^{6}$ (b) 5.13 $\times 10^{6}$ (c) $5.89 \times 10^{6}$ (d) $6.55 \times 10^{6} / \mathrm{ft}$ 
The meanflow for all wind tunnel configurations was obtained by using the LAURA code [5-6]. To develop transition correlations based on algebraic meanflow parameters, laminar flow solutions for several representative configurations with different values of forebody cone angle, unit Reynolds numbers, and angles of attack had been computed during earlier, experimental investigations at NASA Langley Research Center [1]. These meanflow solutions computed are used for stability based transition analyses in this paper. A multi-block structured mesh is used for all meanflow computations. Figure 4 shows the six-block, half a million grid points, structured mesh and the computed pressure contours on both the vehicle surface and the symmetry meridian. The computational domain and grid have been adapted to the bow shock for better accuracy and performance. The computed laminar surface heating distribution in Fig. 4(c) qualitatively resembles that shown in Fig. 3(a), indicating reasonably good agreement with measured data using this mesh. In most of the meanflow computations, there are only 64 points along the wall-normal direction with most of them clustered very close to the wall to resolve the near wall temperature gradient and surface heating rate prediction. While such mesh is adequate for surface heat transfer predictions, it is in generally insufficient to resolve the entire boundary layer, which is more important than surface heat transfer for stability calculations.

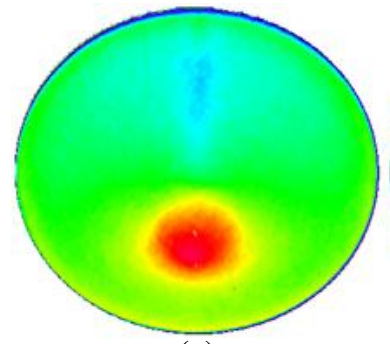

(a)

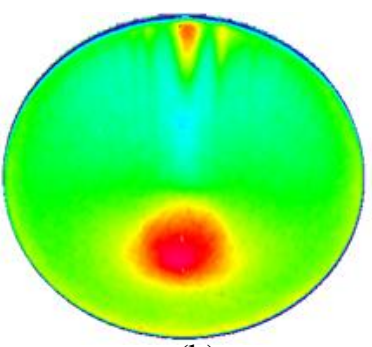

(b)

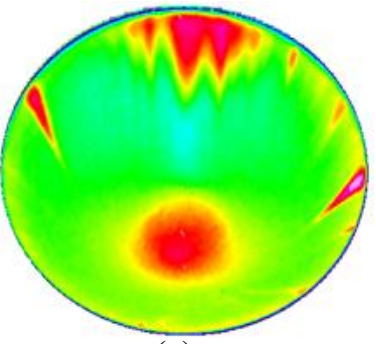

(c)

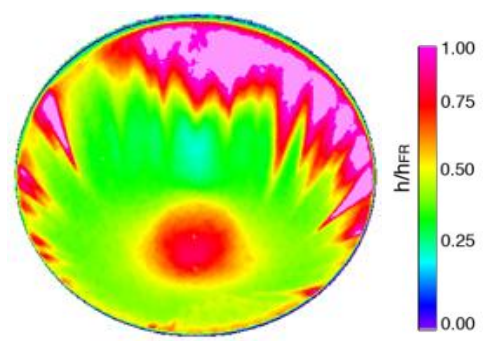

(d)

Figure 3 Distribution of surface heating levels from experimental thermographic images for 60-deg spherecone wind tunnel models at 16 degree angle of attack and four unit Reynolds numbers: (a) $3.95 \times 10^{6}$ (b) 5.13 $\times 10^{6}$ (c) $5.89 \times 10^{6}$ (d) $6.55 \times 10^{6} / \mathrm{ft}$

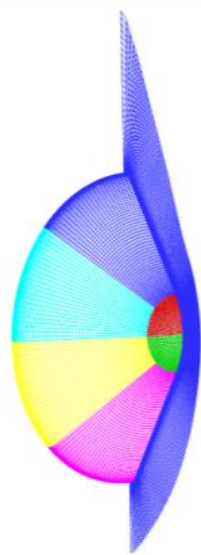

(a)

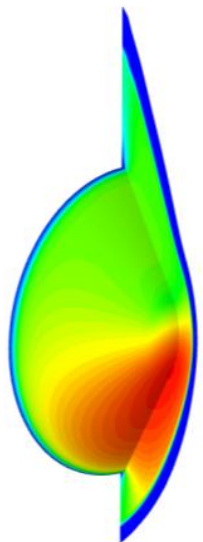

(b)

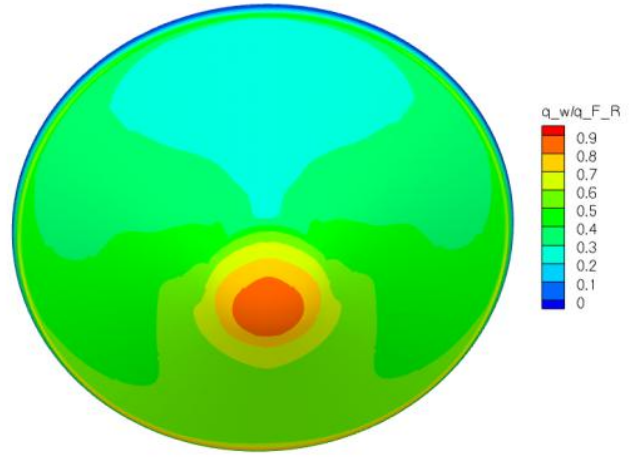

(c)

Figure 4 Mesh and computed pressure and laminar surface heat flux contours for a Mach 6 flow past a 60-deg spherecone wind-tunnel model of the MSL configuration at 16-deg angle of attack and unit Reynolds number of $3.59 \times 10^{6}$, showing (a) multi-block strucutred mesh (b) pressure distribution (c) normalized surface heat flux distribution with respect to peak (stagnation) value.

\section{Stability Calculations and Meanflow Grid-Convergence Study}

Stability calculations have been performed by the Langley Stability and Transition Analysis Codes (LASTRAC) [78]. The LASTRAC software is a suite of general purpose, physics-based, stability and transition analysis tools that encompass a broad range of state-of-the-art numerical methods based on linear stability theory, linear and nonlinear parabolized stability equations (PSE), and adjoint PSE [9]. Stability calculations and N-factor correlations can be performed for both 2D/axisymmetric and general 3D configurations [10]. Nonlinear PSE modules in LASTRAC have been used to study crossflow transition and for cross-validation against DNS computations [11-12]. 
For stability calculations, the meanflow obtained by LAURA is interpolated to a one-block, 401x201 surface mesh shown in Fig. 5(a) (for the 60-deg sphere-cone model). Computed meanflows are interpolated using bi-linear and cubic-spline polynomials along the surface and the wall-normal directions, respectively. Typically, about 101 to 201 wall-normal points are used in the stability calculations to ensure grid independent eigenvalue or PSE solutions. Under-resolved meanflows require, in general, more points for converged stability solutions. LASTRAC provides several options for 3D boundary layers. In this paper, extensive calculations were performed along the symmetry axis $(y=0)$ for first-mode type disturbances and streamlines paths are used for crossflow instability $\mathrm{N}$-factor calculations. Figure 5(b) shows a typical inviscid streamline pattern for the same 60-deg sphere-cone model. For all the configurations presented hereafter, stability calculations were presented only for the leeward side. Calculations on the windward side surface for all three models at experimental conditions did not reveal any unstable modes.
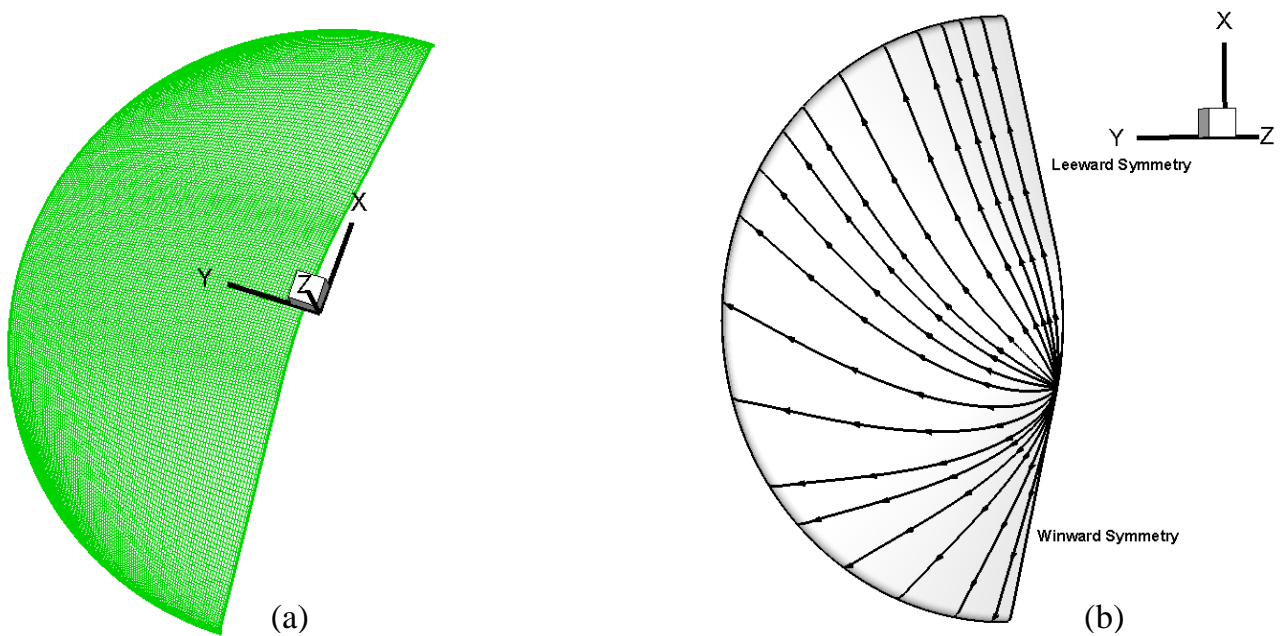

Figure 5 Surface mesh and inviscid streamline patterns used for stability calculations for the 60-deg spherecone model: (a) 401x201 surface mesh (only showing every other points) (b) inviscid streamlines

As mentioned previously, meanflow computations in ref.[1] were performed with grids designed to resolve surface heating predictions. The grid adaption procedure in LAURA is designed to improve the numerical accuracy of the predicted surface heat transfer rate by packing a sufficient number of grid points very close to the viscous wall. Although new mean flow calculations were beyond the scope of this initial effort, the impact of inaccuracies/uncertainty in the mean flow profiles on the stability predictions was evaluated via a limited grid convergence study for one of the configurations, namely, the 60-deg sphere-cone model at $\alpha=16^{0}$ and $\operatorname{Re}=$ $5.89 \times 10^{6} / \mathrm{ft}$. Figure 6 shows the computed meanflow profiles at one representative location downstream of the stagnation point along the symmetric meridian. Four different wall-normal grids are used: 64 points, 128 points, 256 points, and 256 points redistributed by improving the grid density near the boundary-layer edge. Among all the wall-normal points, there are about 33, 66, 128, and 104 points inside the boundary layer, respectively. The first three grids are the typical grids used by LAURA for surface heat transfer predictions and the last one is slightly modified by moving some points away from the wall (see Fig. 6(a)). Grid adaptation was used for the first three grids, but not the last one. All four grids have a substantial number of points very near the wall. While the nearwall solution is very well-converged, solutions near and beyond the boundary layer edge appear to be changing with all four grids. A relevant quantity to evaluate meanflow quality for stability calculation is the general inflectional quantity defined as $g i=d(\rho d u / d y) / d y$ (shown in Fig. 6(c)). A zero in this profile corresponds to the critical layer of a neutrally stable mode according to the inviscid stability theory. While the typical mesh used by LAURA (with adaptation) provides good resolution for the surface heat transfer, it does not adequately resolve the outer part of the boundary layer. It was verified, however, that the outer resolution as measured by gi(y) profiles derived from the interpolated mean flow for the last two grids has approached visual convergence. It was also found that the extremely fine near-wall grid in the adapted grid mean flow profiles actually contributes to increased noise in the $g i(y)$ profile. These oscillations are confined very near the wall and do not have a major effect on stability calculations. 
Stability calculations were performed for the above four different grids and results are shown in Fig. 7 for $\mathrm{N}$ factors of various first-mode instability waves with different frequencies versus $x / R$ ( with $x=0$ at the sphere-cone center and $\mathrm{R}$ the base radius). As expected, computed $\mathrm{N}$ factors are not fully converged with respect to meanflow grids. However, stability solutions for the two finest grids are already quite close (with an overall difference in $\mathrm{N}$ values of about 0.2 ). The overall scatter of $\mathrm{N}$ values for various grid resolutions is about 1.1. Note that a grid-converged meanflow solution required for stability calculations does not necessarily take more than 256 points. Based on past experience, a properly distributed wall-normal grid with only 40 50 points within the boundary layer may give better stability results than a finer grid with very strong clustering only near the wall. The above comparison indicates that most of the meanflows from ref. [1] for various MSL configurations with 64 wall normal points are unlikely to provide a highly grid-converged basic-state for the purpose of stability calculations. Nevertheless, a broad parameter study based the available mean flow solutions was deemed to be a useful exercise for two reasons, namely, to delineate trends with respect to relevant geometric and flow parameters, and to provide a foundation for a refined set of computations with improved mean flow solutions. Both of these issues are addressed in the following section of this paper.

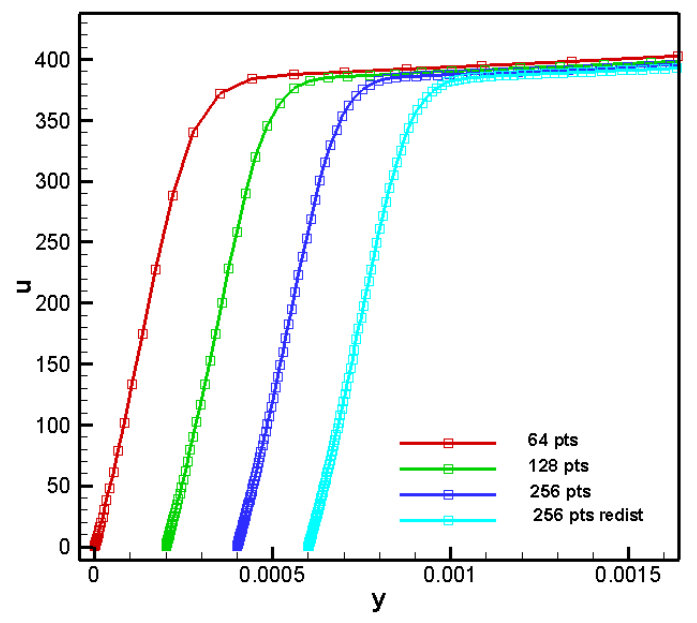

(a)

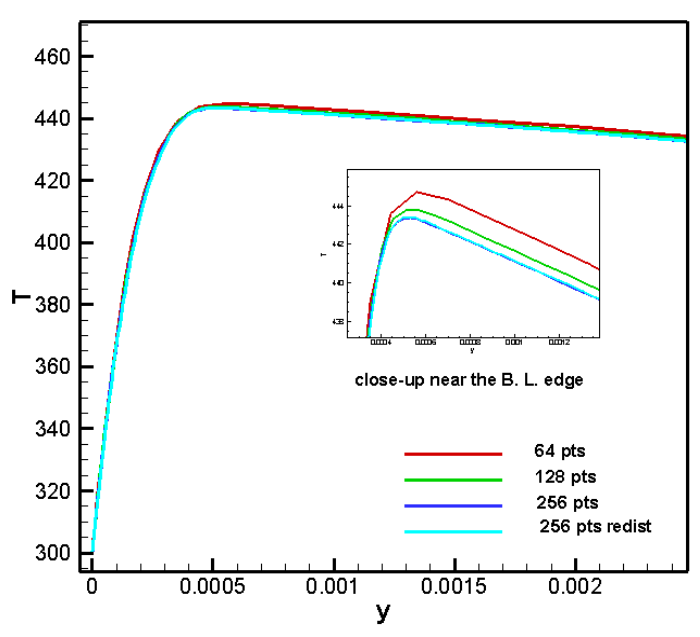

(b)

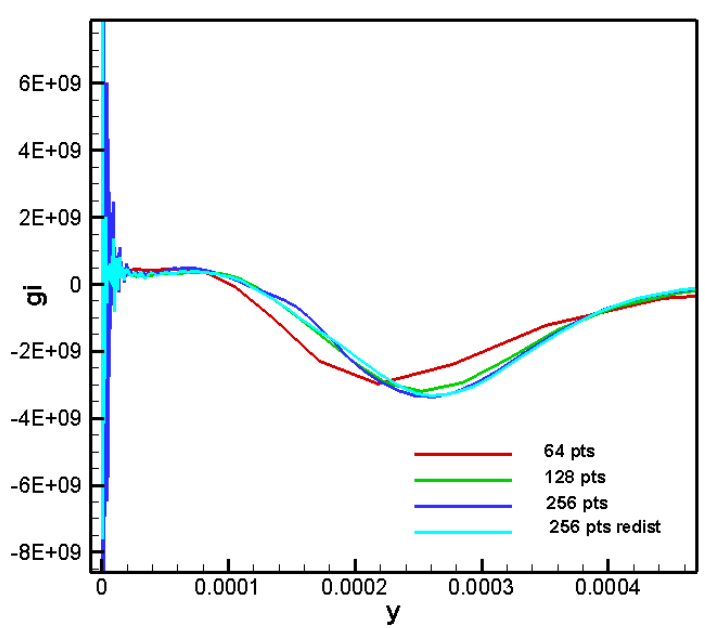

(c)

Figure 6 Representative velocity, temperature and general inflectional quantity profiles versus wall-normal distance (in meters) at the symmetry plane for the 60-deg sphere-cone model: (a) streamwise velocity and grid points (y shifted artificially for clarity) (b) temperature (c) general inflectional quantity 


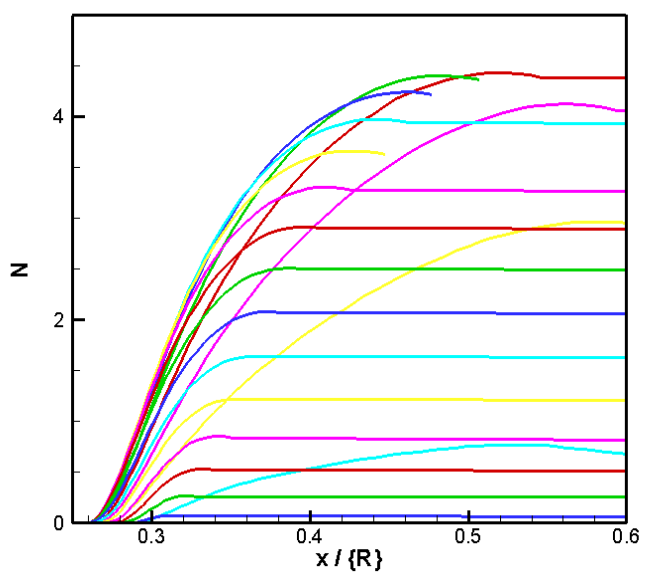

(a)

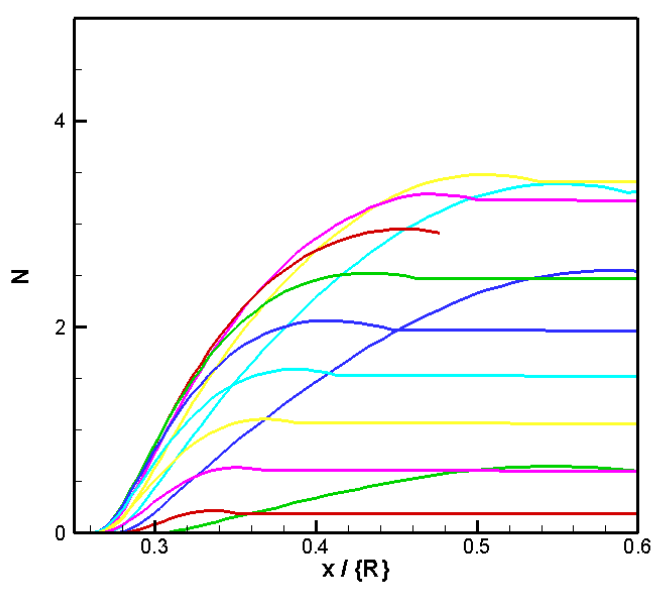

(c)

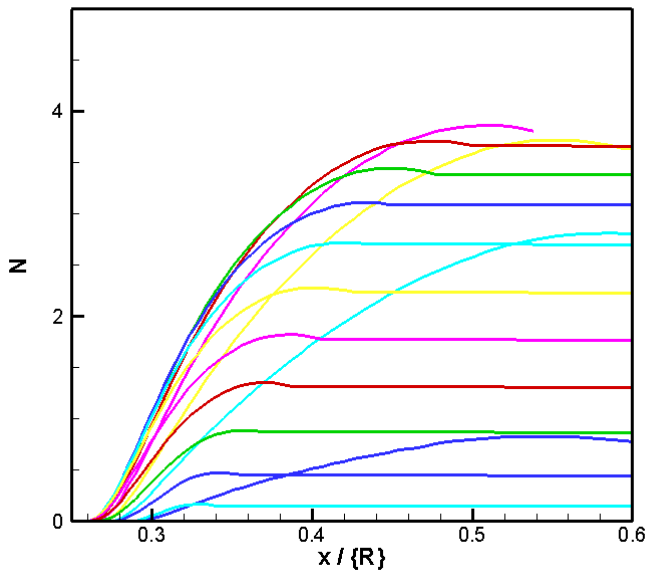

(b)

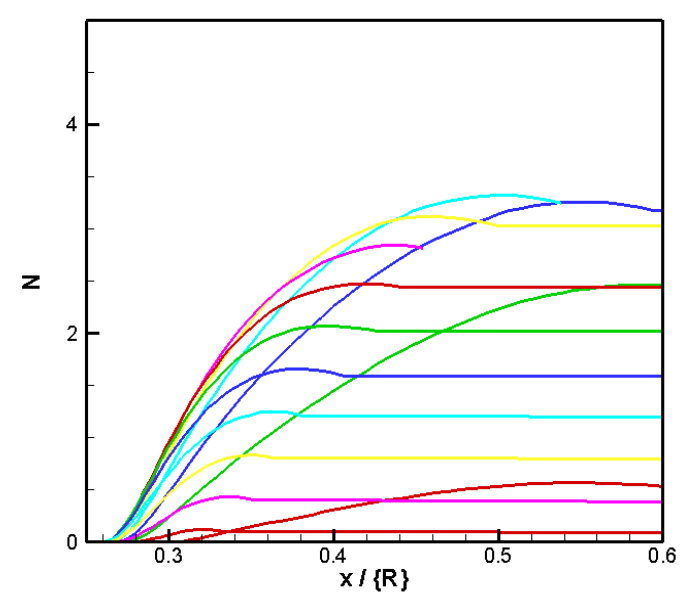

(d)

Figure 7 Computed first-mode $\mathrm{N}$-factor distributions along the symmetry meridian for various disturbance frequencies using four different meanflow grids : (a) 64 points (b) 128 points (c) 256 points (d) 256 points redistributed

\section{Results and Discussion}

$\mathrm{N}$-factor calculations were performed for most of the experimental configurations presented in ref.[1]. Before showing the details, we examine the representative inviscid flow characteristics on the model surface in Fig. 8 where Mach number distributions well outside the boundary layer edge are plotted. Along the symmetric meridian, the flow is accelerated from subsonic on the windward side to supersonic on the leeward side. Smaller cone angle results in stronger acceleration, thus the 50-deg cases reach a peak Mach number of about 2.5, as compared to 1.7 and 1.4 for 60 -deg and 70-deg sphere-cone models, respectively. First mode instability waves are expected to be the dominant stability mechanism on the symmetry plane at this Mach number range. Except for the 50-deg case, 2D first modes are the most-amplified instability waves due to the low supersonic speeds. 

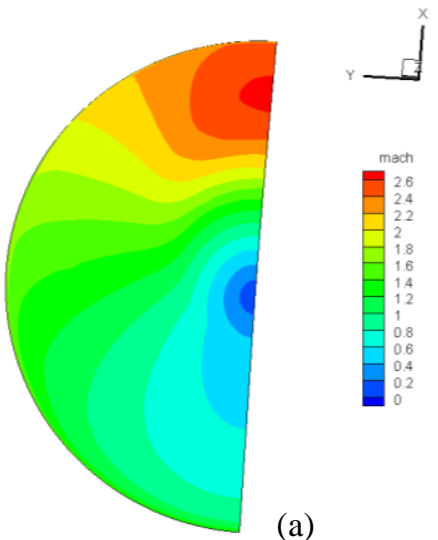

(a)
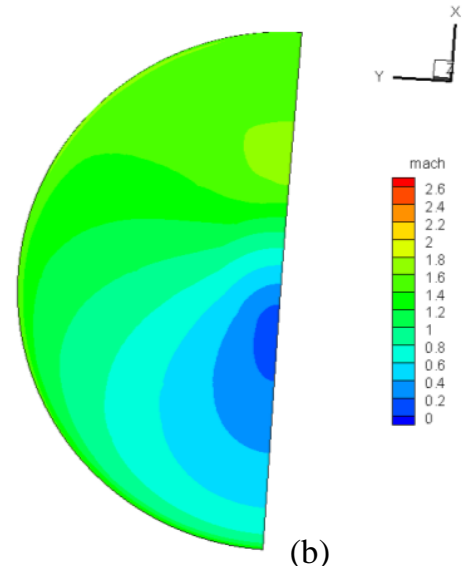

(b)
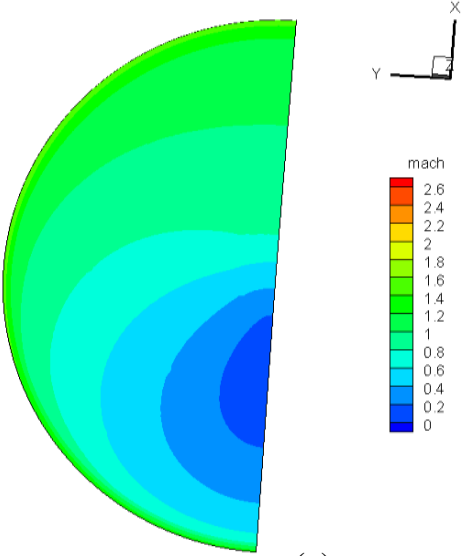

(c)

Figure 8 Inviscid Mach number distributions for three representative configurations at 16-degree angle of attack and $\mathrm{Re}=6.6 \times 10^{6} / \mathrm{ft}$ : (a) 50-degree (b) 60-deg (c) 70-deg sphere-cone models

Accurately calculating the crossflow Reynolds number is difficult for under-resolved meanflow because of the poor resolution near the boundary layer edge. To assess possible crossflow instability on the model surface, the estimated crossflow Reynolds number contours are plotted in Fig. 9 for the same three representative cases. The non-smooth contours indicate uncertainties in the edge estimates. Nevertheless, they provide a means for estimating possible crossflow instability modes. Among three cases, the 70-deg case shows very minimum crossflow. The strength of crossflow increases for the 50-deg and 60-deg cases. These observations seem to be in agreement with experimental transition onsets depicted in Figs. 2(d) and 3(d) for the 50-deg and 60-deg models under the same conditions. Both show away from symmetry meridian transition fronts with the 50-deg case being more unstable. Note that transition fronts appear to be jagged in these experimental thermographic images, possibly due to surface imperfections of the model. We discuss both first-mode and crossflow instability mechanisms separately below.

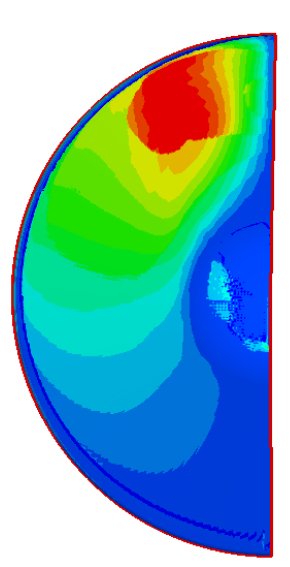

(a)
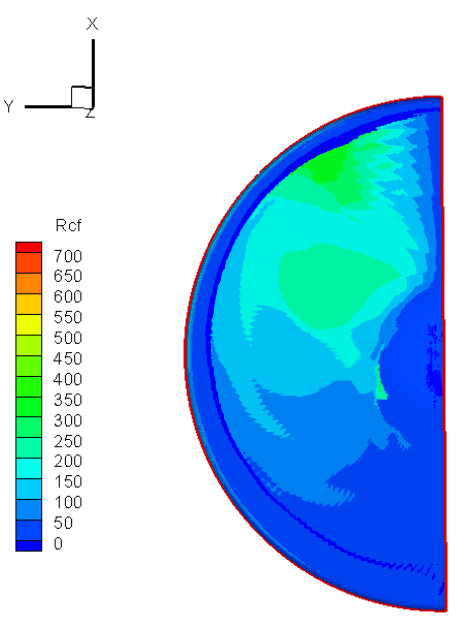

(b)

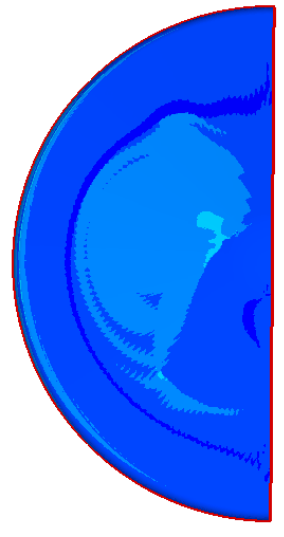

(c)

Figure 9 Estimated crossflow Reynolds number distributions for three representative configurations at 16degree angle of attack and $\operatorname{Re}=6.6 \times 10^{6} / \mathrm{ft}:$ (a) 50-degree (b) 60-deg (c) 70-deg sphere-cone models

\section{First-Mode Instability along the Leeward Line of Symmetry}

Experimentally measured surface heat flux distributions along the symmetry meridian are available for all three sphere-cone models from ref. [1]. As shown in Fig. 10, transition onset is indicated by a sudden rise in heat flux in the vicinity of $x / R=0.5$ (where $R$ is the base radius of the sphere-cone) for five different unit Reynolds numbers investigated in the experiments. The precise transition onset location varies depending on the Reynolds number. A 
rough estimate gives transition locations at $x / R \approx 0.8,0.6,0.5,0.43$, and 0.4 for unit Reynolds numbers of $4.0 \times 10^{6}$, $5.1 \times 10^{6}, 5.9 \times 10^{6}, 6.6 \times 10^{6}, 7.3 \times 10^{6} / \mathrm{ft}$, respectively.

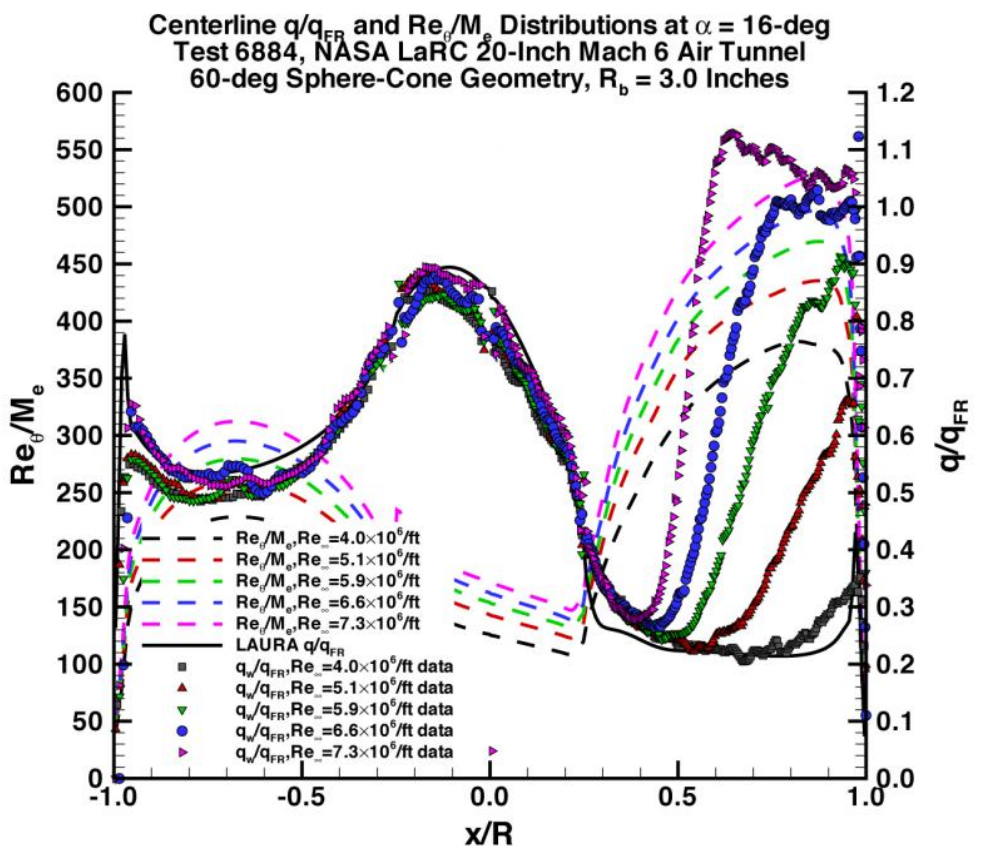

Figure 10 Experimentally measured surface heating distribution for the 60-deg sphere-cone model at 16 degree angle of attack (taken from Fig. 5 of Ref. [1]).

Quasi-parallel linear stability N-factors with all curvature terms included (QPWC) were calculated by maximizing the growth rates for a fixed disturbance frequency at each location for all configurations shown in Fig. 10. The results for various disturbance frequencies are shown in Fig. 11 for all five unit Reynolds numbers. The onset of instability occurs near $x / R=0.25$ for all cases. Most instability modes are rapidly amplified to their peak $\mathrm{N}$-factor near $x / R=0.5$. The most unstable disturbance frequency is around $50-70 \mathrm{kHz}$ for all unit Reynolds numbers. At this low supersonic Mach number, $\mathrm{N}$-factor envelopes formed by 2D first mode waves give only slightly smaller $\mathrm{N}$ factors (less by approximately 0.2). For the two lowest Reynolds number cases, $\mathrm{N}$ values peak at 2.55 and 3.45 around $x / R=0.5$, respectively, then begin to decay beyond the upper-branch neutral stability point (where disturbances reach a maximum value) until the transition location. Such low $\mathrm{N}$ values and the accompanying decaying region may not be sufficient to provide enough amplification for instability growth and transition onset. Nevertheless, strong receptivity and secondary instability at large disturbance amplitude may still cause transition. In contrast, transition takes place within the unstable region for the other three Reynolds numbers. $\mathrm{N}$-factor values at the transition locations inferred from the surface heating distributions are approximately 4, 3.9, and 4.2 for unit Reynolds numbers of $5.9 \times 10^{6}, 6.6 \times 10^{6}, 7.3 \times 10^{6} / \mathrm{ft}$, respectively.

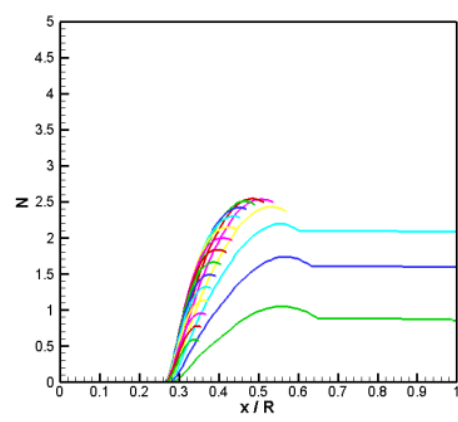

(a)

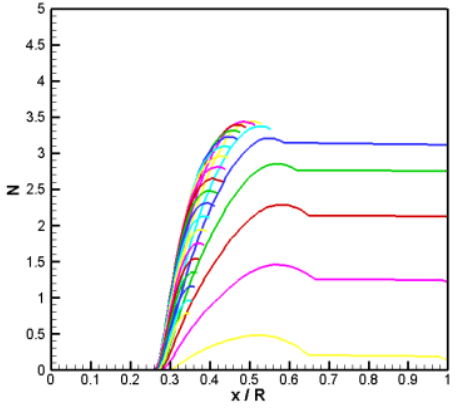

(b)

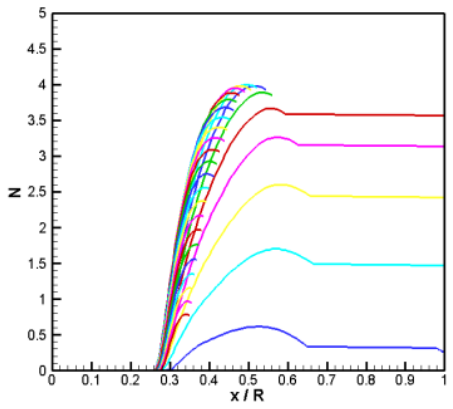

(c) 


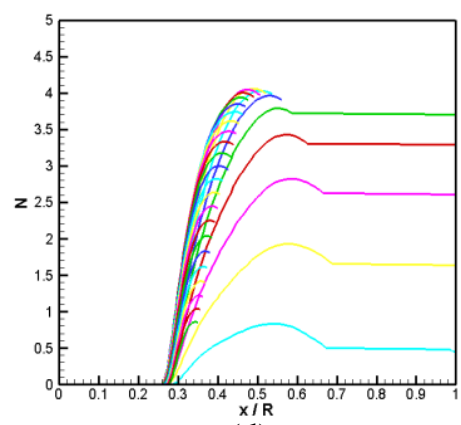

(d)

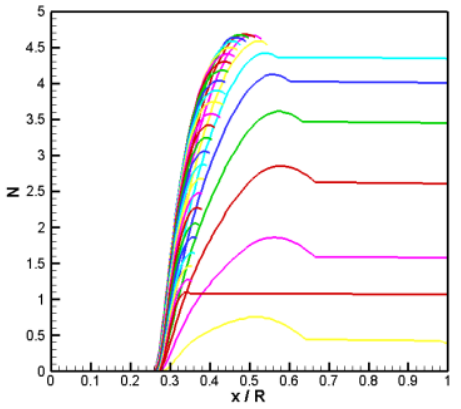

(e)

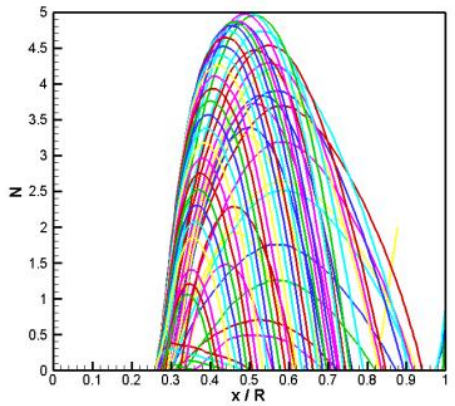

(f)

Figure 11 First-mode $\mathbf{N}$-factor versus $\mathbf{x} / \mathbf{R}$ for various disturbance frequencies along the symmetry axis for 60 deg sphere-cone model at 16 degree angle of attack, QPWC for a-e, linear PSE for f: (a) $3.95 \times 10^{6}$ (b) $5.13 \mathrm{x}$ $10^{6}$ (c) $5.89 \times 10^{6}$ (d) $6.55 \times 10^{6}$ (e) $7.33 \times 10^{6}$ (f) $7.33 \times 10^{6} / \mathrm{ft}$

To assess the non-parallel effect, linear PSE calculations were also performed. Figure 11(f) shows an example of the $\mathrm{N}$ values for various dominant oblique first modes computed for a unit Reynolds number of $7.33 \times 10^{6} / \mathrm{ft}$. In general, the correlating $\mathrm{N}$ value increases slightly. Transition $\mathrm{N}$ values using linear PSE were found to be 4.34, 4.15 , and 4.51 for unit Reynolds numbers of $5.9 \times 10^{6}, 6.6 \times 10^{6}, 7.3 \times 10^{6} / \mathrm{ft}$, respectively; only slightly higher than the corresponding values based on quasi-parallel theory. We also note that because the maximum $\mathrm{N}$-factor values over the length of the leeward meridian are also small (less than 5), transition under these conditions would have been unlikely to happen either in a quiet facility or in a flight disturbance environment.

Effects of the angle of attack can be evaluated from Fig. 12 where the computed $\mathrm{N}$-factor distributions for the same 60-deg sphere-cone model with two different $\alpha$ values of $11^{\circ}$ and $20^{\circ}$ along with a comparison of Mach number distributions for all three angles of attack are shown. Peak $\mathrm{N}$ values are only slightly more than 1 for the smallest angle of attack case. The peak values for the $\alpha=16^{0}$ case (see Fig. 11(c)) are in fact slightly larger than those for the $\alpha=20^{\circ}$ case shown in Fig. 12(b). This stabilizing trend at larger angle of attack may be attributed to the fact that the negative pressure gradient region takes place earlier and coincides with the unstable region of the disturbances for the $\alpha=16^{0}$ case (see Fig. 12(c)). The same conclusions can be drawn for the other unit Reynolds numbers. Experimental data also confirms this general trend; namely, transition onset takes place further downstream on the leeward side symmetry meridian for the $\alpha=20^{\circ}$ cases.

To examine the effect of different sphere-cone models at the same wind tunnel conditions, computed $\mathrm{N}$-factor distributions for all three models at a unit Reynolds number of $7.33 \times 10^{6} / \mathrm{ft}$ and $\alpha=20^{0}$ are plotted in Fig. 13. Firstmode disturbances grow earlier at larger cone angles, achieving their peak $\mathrm{N}$-factor over a short distance. However, disturbances at smaller cone angles tend to be amplified more, resulting in larger values of peak $\mathrm{N}$-factor. Experimental data indicates transition onset at $x / R=0.75,0.48$ for the 50-deg and 60-deg sphere-cone models, respectively. No data is available for the 70-deg model at this unit Reynolds number. However, transition occurs at $x / R=0.28$ for the 70 -deg model at $\mathrm{Re}=6.55 \times 10^{6} / \mathrm{ft}$. Thus transition for the highest Reynolds number should be at $x / R<0.28$ where the $\mathrm{N}$ factors already peak around 1.5 and decay. On the other hand, transition $\mathrm{N}$ values are about 2.93 and 4.45 for the 50-deg and 60-deg models, respectively. 


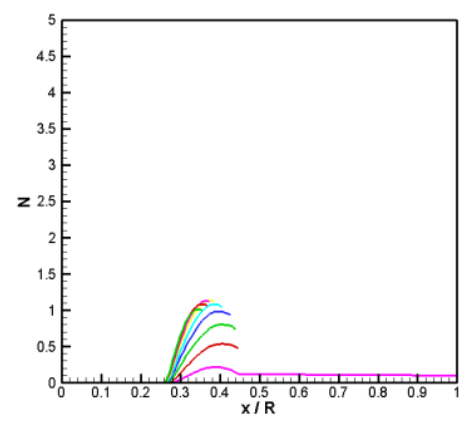

(a)

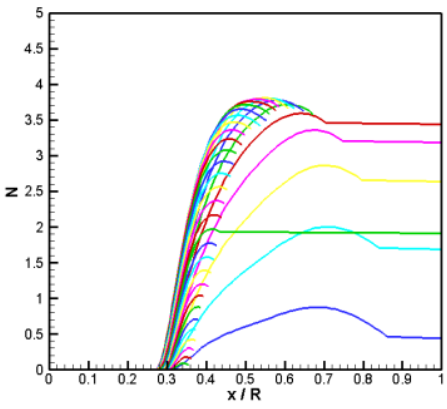

(b)

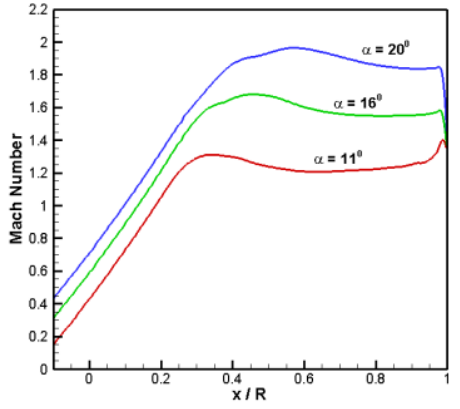

(c)

Figure 12 Comparison of first-mode $N$ factors for the $60-\mathrm{deg}$ sphere cone model at $\operatorname{Re}=5.89 \times 10^{6} / \mathrm{ft}$ at $\mathrm{two}$ different angles of attack: (a) $\alpha=11^{\circ}$ (b) $\alpha=20^{\circ}$ (c) Mach number distributions for all three angles of attack.

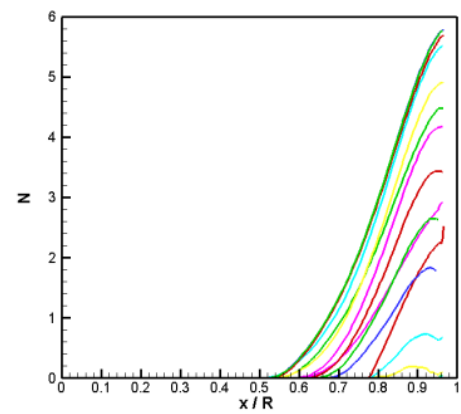

(a)

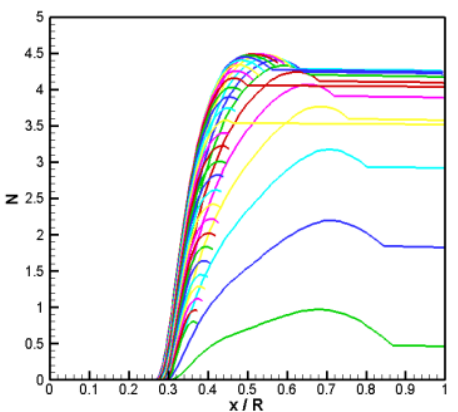

(b)

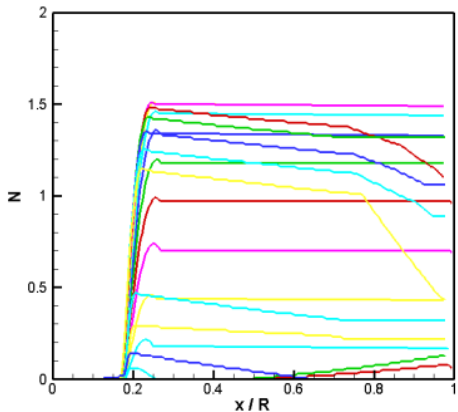

(c)

Figure 13 Comparison of first-mode $\mathrm{N}$ factors at $\mathrm{Re}=7.33 \times 10^{6} / \mathrm{ft}$ for all three sphere-cone models at $\alpha=20^{0}$ : (a) 50-deg (b) 60-deg (c) 70-deg.

Results shown in Fig. 13 are also representative for the three different sphere-cone models under different wind tunnel conditions. The 50-deg model has, in general, strong disturbance amplification close to the edge of the model surface along the symmetry meridian. In contrast, disturbances quickly grow to their peaks near $x / R=0.5$ for the 60-deg model under all wind tunnel conditions. All cases computed for the 70-deg model have an $\mathrm{N}$-factor distribution similar to that shown in Fig. 13(c). Disturbances grow at very small $x / R$ and no additional instability modes were found beyond that. The peak $\mathrm{N}$ values decrease as the unit Reynolds number decreases. The value of 1.5 shown in the figure is the largest $\mathrm{N}$ value for the 70-deg model. Despite the small $\mathrm{N}$ values, transition is still observed in the experiments for this model at most of the wind tunnel conditions with $\alpha=16^{\circ}$ and $\alpha=20^{\circ}$. Further studies with a more refined meanflow are necessary to understand the cause of such low $\mathrm{N}$-value for first-mode transition for the 70-deg model.

Figure 14 summarizes the correlated transition $\mathrm{N}$ values for most cases with a clearly discernable experimental transition onset along the symmetry meridian. All cases with a peak $\mathrm{N}$ factor well upstream of the measured transition location (similar to that shown in Fig. 13(c)) are excluded in the figure. The transition $\mathrm{N}$ value falls between 3.5 and 4.5. In general, transition $\mathrm{N}$-factor using linear PSE is only slightly higher than the QPWC results. The "average" transition $\mathrm{N}$ value for this series of experiments appears to be around 4 . It is more interesting to note that except for one outlier, transition $\mathrm{N}$ value scales roughly linearly with the wind tunnel unit Reynolds number. Such linear variation implies strong receptivity effects under a noisy environment. Receptivity to different noise spectra along the wind tunnel wall under different reservoir conditions causes transition Reynolds number to increase with unit Reynolds numbers for conventional wind tunnels [16]. In contrast, the experiments performed for a flat plate and a cone at NASA Langley's Mach 3.5 quiet tunnel facilities [16] show a negligible unit Reynolds number effect. Different transition Reynolds number results in a varying transition $\mathrm{N}$-factor under different wind tunnel conditions for the first-mode transition shown here. These relatively small transition $\mathrm{N}$ factors are apparently in line with previous transition studies for a flared cone experiment performed in the same 20-inch Mach 6 tunnel [13]. Transition in that particular experiment was dominated by second mode waves instead, but the correlating $\mathrm{N}$ factors were again found to be approximately 3.7 as opposed to 8 or so for the same model tested in a quiet facility 
at the same Mach number. In general, transition $\mathrm{N}$ factors have been found to be significantly smaller when transition onset is measured within a conventional facility.

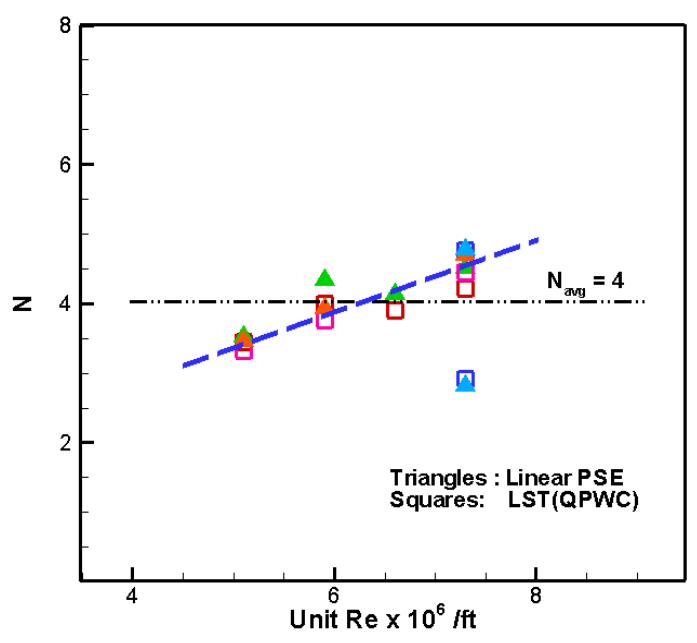

Figure 14 Correlated Transition N-factor computed by LST and PSE versus unit Reynolds numbers for all cases computed.

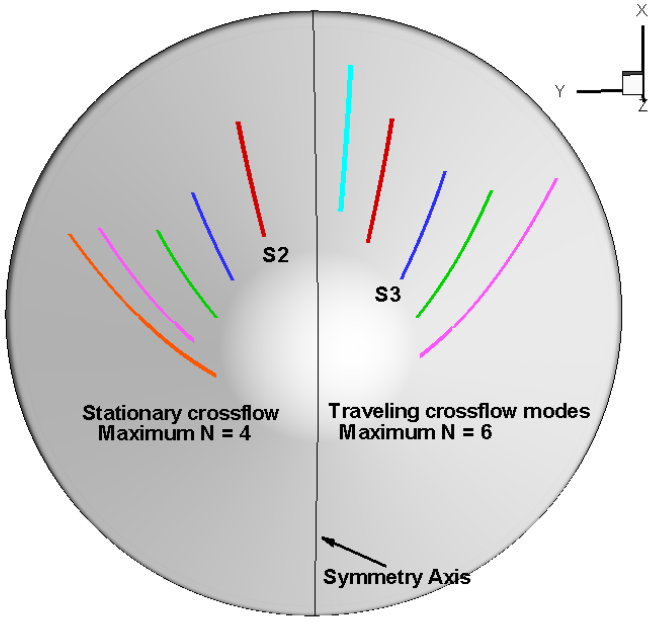

Figure 15 Predicted transition fronts by stationary cases and traveling crossflow instability with $N$ values of 4 and 6 , respectively for the 50-deg model at $\operatorname{Re}=6.6 \times 10^{6}$ and $\alpha=16^{0}$.

\section{Crossflow Instability away from the Leeward Line of Symmetry}

Investigations of possible crossflow instability induced transition were performed for two selected cases. The first configuration corresponds to the case shown in Fig. 2(d). The corresponding Mach number and estimated crossflow Reynolds number contours are shown in Figs. 8(a) and 9(a), respectively. Although not presented here, computed first-mode $\mathrm{N}$ factors are similar to those depicted in Fig. 13(a). Disturbance amplification happens rather late, near the edge of the model and the $\mathrm{N}$ values reach about 6.7 at $x / R=0.9$ where experimental data indicates transition [1]. The envelope method is used for $\mathrm{N}$-factor integration along a set of predetermined streamlines as shown in Fig. 15. Disturbance growth rate is maximized at each location and integrated downstream along the streamline marching path. Each streamline shown in the figure starts at the onset of instability and extends to the location where a prescribed $\mathrm{N}$ value (4 for stationary crossflow and 6 for traveling crossflow instability modes) has been reached. The selection of 4 and 6 is based on the correlated stationary and traveling crossflow transition $\mathrm{N}$-factor of the HIFiRE-5 vehicle model tested in the same NASA Langley wind tunnel [15]. The predicted transition fronts agree reasonably well with the off-axis transition patterns shown in Fig. 2(d). Figures 16(a) and 16(b) depict the N-factor distributions for two of the streamline paths S2 and S3 shown in Fig. 15, which have the most disturbance growth. The overall $\mathrm{N}$-factor values reach about 8.2 and 10 along S3 for stationary and traveling crossflow instability waves, respectively. The most amplified traveling modes are around $15 \sim 20 \mathrm{kHz}$. Maximum $\mathrm{N}$ values are smaller along other streamlines. Due to the relatively small $\mathrm{N}$-factor along most streamlines, it is likely that transition would not occur or takes place very near the cone edge under a quiet environment such as flight.

Crossflow stability calculations were also performed for the 60-deg sphere-cone model at $\operatorname{Re}=5.89 \times 10^{6}$ and $\alpha=16^{0}$. As already discussed, transition along the leeward axis occurs at $x / R=0.5$ with a transition $\mathrm{N}$ value of about 4 (see Figs. 10 and 11(c)). Only very weak stationary crossflow instability with almost negligible $\mathrm{N}$ values were identified over the entire surface. Traveling crossflow waves were found to be unstable over several streamline paths similar to those shown in Fig. 15 for the 50-deg model. However, the overall N-factor only reaches about 2.2. Figure 16(c) shows $\mathrm{N}$-factors along one representative streamline integration path for this model. It is apparently much more stable to crossflow modes than the previously discussed 50-deg case. The experimental thermographic image shown in Fig. 3(c) also confirms that the only visible transition fronts as evident by a rapid increase in surface heating occur along the leeward line of symmetry. 


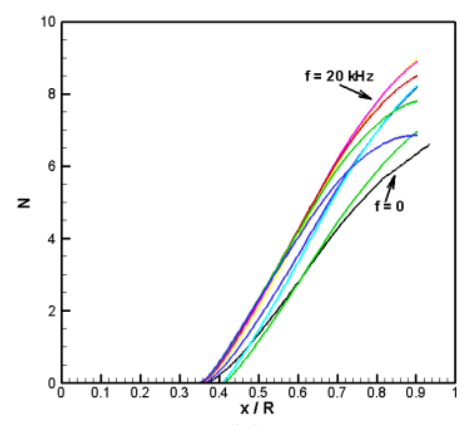

(a)

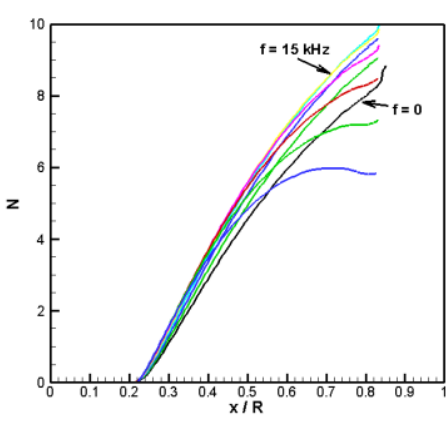

(b)

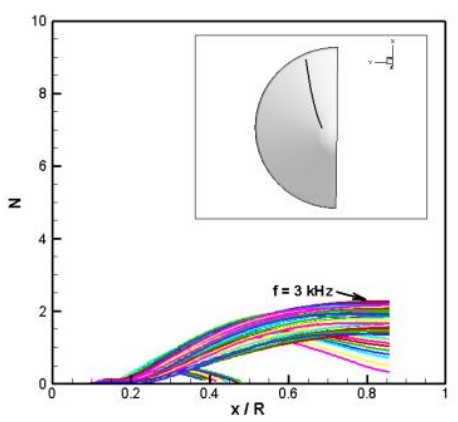

(c)

Figure 16 Computed stationary and traveling crossflow instability $\mathbf{N}$ facotrs along various streamline paths: (a) path S2 shown in Fig. 15 (b) path S3 shown in Fig. 15 (c) 60 -deg model at $\operatorname{Re}=5.89 \times 10^{6} / \mathrm{ft}^{\mathrm{and}} \alpha=16^{0}$, corresponds to Fig. 3(c).

\section{Summary}

Transition analyses were performed using the LASTRAC software for three wind tunnel models of the MSL vehicle configurations with 50-deg, 60-deg, and 70-deg sphere-cone geometries at several angles of attack and wind tunnel conditions. The dominant instability mechanism at the symmetry median was found to be of the first-mode type. Similar to previous transition correlations based on second mode transition within the same facility (NASA Langley's 20-inch Mach 6 wind tunnel), the onset of first-mode transition correlates with an approximate N-factor of 4. The correlated transition $\mathrm{N}$-factor varies roughly linearly with the wind tunnel unit Reynolds number. The 70deg wind tunnel model was found to be more stable to first-mode disturbances than the 50-deg and 60-deg models. Detailed stability calculations were carried out for grid-convergence studies related to meanflow computations and improved grid distributions were suggested for the next phase of this research. Stability calculations for both stationary and traveling crossflow instability waves were also performed for selected configurations. The results suggest that a transition $\mathrm{N}$ value of 4 and 6 for stationary and traveling crossflow modes, respectively, appears to give reasonable transition estimates off the leeward meridian of symmetry. These results are in line with a slender cone type configuration investigated within the same wind tunnel [15]. This paper provides preliminary analyses for transition over the wind tunnel models relevant to the MSL flight vehicle. The findings are sufficiently encouraging to warrant a full-fledged transition analysis based on mean flow computations that are geared specifically for predictions of instability wave propagation. With more accurate mean flows obtained based on the refined mesh suggested in this study, physics-based transition prediction could be used in the future to aid the aerodynamic and aerothermodynamic design for blunt body configurations similar to the MSL entry vehicle . The follow-on component of this ongoing study will also include numerical simulations to identify unique aspects of receptivity to both free-stream disturbances and surface irregularities in the context of such blunt vehicles.

\section{References}

1. Hollis, B. R., Liechty, D. S., Wright, M. M., Holden, M. S., Wadhams, T. P., MacLean, M., and Dyakonov, A., "Transition Oset and Turbulent Heating Measuements for the Mars Science Laboratory Entry Vehicle," AIAA Paper 2005-1437, 2005.

2. Lockwood, M. K., Sutton, K., Prabhu, R., Powell, R. W., Graves, C. A., Epp, C., and Carman, G. L., "Entry Configurations and Performance Comparisons for the Mars Smart Lander", AIAA Paper 2002-4407, August 2002.

3. Hollis, B. R. and Liechty, D. S., "Boundary-Layer Transition Correlations and Aeroheating Predictions for Mars Smart Lander," AIAA Paper 2002-2745, August, 2002.

4. Liechty, D. S. and Hollis, B. R., "Heat Shield Parametric Experimental Aeroheating for a Mars Smart Lander," AIAA Paper 2002-2746, August 2002.

5. Gnoffo, P. A., "An Upwind-Biased, Point-Implicit Algorithm for Viscous, Compressible Perfect-Gas Flows," NASA TP-2953, February 1990.

6. Cheatwood, F. M., and Gnoffo, P. A., "User's Manual for the Langley Aerothermodynamic Upwind Relaxation Algorithm (LAURA),” NASA TM 4674, April, 1996. 
7. Chang, C.-L., "Langley Stability and Transition Analysis Code (LASTRAC) Version 1.2 User Manual," NASA/TM-2004-213233, June, 2004.

8. Chang, C.-L., "The Langley Stability and Transition Analysis Code (LASTRAC): LST, Linear and Nonlinear PSE for 2-D, Axisymmetric, and Infinite Swept Wing Boundary Layers," AIAA Paper 20030974, 2003.

9. Chang, C.-L. and Choudhari, M. M., "Boundary-Layer Receptivity and Integrated Transition Prediction," AIAA Paper 2005-0526, 2005.

10. Chang, C.-L., "LASTRAC.3d: Transition Prediction in 3D Boundary Layers," AIAA Paper 2004-2542, Portland, Oregon, Jun. 2004.

11. Jiang, L., Chang, C.-L., Choudhari, M., and Liu, C., "Instability-Wave Propagation in Boundary-Layer Flows at Subsonic through Hypersonic Mach Numbers," Mathematics and Computers in Simulation, Vol. 65, pp. 469-487, 2004.

12. Choudhari, M. M., Chang, C.-L. and Jiang, L., "Towards Transition Modeling for Supersonic Laminar Flow Control," Philosophical Transactions of the Royal Society Series A: Mathematical, Physical and Engineering Sciences, vol. 363, No. 1830, pp. 1041-1259, 2005.

13. Horvath, T. J., Berry, S. A., Hollis, B. R., Chang, C.-L., and Singer, B. A., "Boundary Layer Transition On Slender Cones In Conventional and Low Disturbance Mach 6 Wind Tunnels," AIAA Paper 2002-2743, $32^{\text {nd }}$ AIAA Fluid Dynamics Conference, St. Louis, 2002

14. Johnson, H., Candler, G, and Wright, M., "Boundary Layer Stability Analysis of Mars Science Laboratory Aeroshell," AIAA Paper 2006-920, 2006.

15. Choudhari, M. M., Chang, C.-L., Jentink, T, Li, F., and Berger, K., "Transition Analysis for the HIFiRE-5 Vehicle," AIAA Paper 2009-4056, 2009.

16. Chen, F.-J., Malik, M. R., and Bechwith, I. E., "Boundary-Layer Transition on a Cone and Flat Plate at Mach 3.5," AIAA Journal, vol. 27, No. 6, pp. 687-693, 1989. 